\title{
Convergence Analysis of a New Class of Flexible Hybrid Concatenated Codes
}

\author{
Bin Zhao and Matthew C. Valenti \\ Lane Dept. of Comp. Sci. \& Elect. Eng. \\ West Virginia University \\ Morgantown, WV 26506-6109 \\ email: bzhao@csee.wvu.edu, mvalenti@wvu.edu
}

\begin{abstract}
This paper focuses on a new class of flexible hybrid concatenated codes which are able to achieve any desired bit error rate performance between that of a parallel and serial concatenated convolutional code. The main contribution of this paper is a convergence analysis of the decoder, which is facilitated by an alternative representation of the code structure and an extrinsic information (EXIT) chart. In addition to giving some insight into the behavior and design of this particular type of hybrid code, this paper also provides new insight into the relationship between parallel and serial concatenated codes as well as the hybrid concatenated code proposed by Divsalar and Pollara.
\end{abstract}

Keywords - Turbo codes, hybrid codes, serial concatenated codes, parallel concatenated codes, EXIT chart, iterative decoding convergence analysis.

\section{INTRODUCTION}

In [1], [2], a new class of hybrid concatenated codes were proposed and shown to split the bit error performance of parallel concatenated convolutional codes (PCCCs) and serial concatenated convolutional codes (SCCCs) in both the waterfall and error floor regions. The hybrid code was generated by puncturing the output of a slightly modified parent SCCC encoder comprised of a pair of recursive systematic convolutional (RSC) encoders. The main modification to the SCCC encoder involved an interleaver design which essentially interleaves the systematic and parity bits of the outer code independently. This constraint on the interleaver ensures that the output of the inner encoder can be split into four distinct fields, the global systematic bits (systematic outer / systematic inner $\left(S_{o} / S_{i}\right)$ ), the "single" parity due to only the outer encoder (parity outer / systematic inner $\left.\left(P_{o} / S_{i}\right)\right)$, the single parity due to only the inner encoder (systematic outer / parity inner $\left(S_{o} / P_{i}\right)$ ), and the "double" parity bits (parity outer/parity inner $\left(P_{o} / P_{i}\right)$ ).

It is the presence of the double parity bits that differentiates this SCCC from a PCCC. In particular, puncturing all the double parity bits generates a PCCC code while keeping all of them maintains a SCCC code. Puncturing a fraction of the double parity bits creates a hybrid code whose performance is between that of a PCCC and SCCC code. When half of the bits in both $P_{o} / P_{i}$ and $S_{o} / P_{i}$ fields are deleted, the code operates as a punctured (rate $1 / 3$ ) SCCC, but as more $P_{o} / P_{i}$ bits are punctured (and more

This work was supported by the Office of Naval Research under grant N00014-00-0655.

0-7803-7339-1/02/\$17.00 (C)2002 IEEE
$S_{o} / P_{i}$ bits maintained) the code behaves more like a PCCC. More specifically, as more double parity bits are punctured, the "waterfall" or "turbo cliff" occurs at a lower SNR while the error floor is raised. When all the double parity bits are punctured (and all single parity bits maintained) the code performance is identical to that of an unpunctured rate $1 / 3$ PCCC.

The benefit of this approach is that it gives codes with error performance between the two extremes of PCCC and SCCC codes. Since the hybrid code is created by simply puncturing the output of a SCCC encoder, no additional hardware is required to implement it. In fact, because a PCCC code can be generated by appropriately puncturing a standard SCCC code (with appropriate interleaver), it might be wise for IC designers and manufacturers to focus on SCCC products rather than on PCCCs. However, it should be noted that although a SCCC decoder can be used to decode a PCCC code, the complexity of the SCCC decoder is 1.5 times that of the PCCC decoder since the inner code is clocked at twice the rate of the outer code. Furthermore, the proposed code structure lends itself to application in hybrid FEC/ARQ sytemes [1].

A hybrid concatenated coding scheme was previously proposed in [3]. This technique, termed hybrid concatenated convolutional codes (HCCCs), involves a convolutional code concatenated in parallel with a SCCC. A union bounding analysis showed that HCCCs perform better than both SCCCs and PCCCs in both the waterfall region and error floor. While our proposed code does not simultaneously perform better than both PCCCs and SCCCs, its complexity is much lower. In fact, we show later that the proposed code is a subclass of the HCCC of [3].

\section{A Class of Flexible Hybrid Codes}

The proposed hybrid encoder is shown in Fig. 1 and is composed of two major parts: a rate $1 / 4$ parent SCCC and a puncturing unit. The parent SCCC has identical inner and outer encoders separated by a structured interleaver. Both constituent codes are recursive systematic convolutional (RSC) codes with the same generator $(35,23)$. The structured interleaver is designed such that it will map the systematic bits into the first half of the interleaved frame and parity bits into the second half. This is equivalent to interleaving the systematic and parity bits independently and then putting them in cascade so that all the interleaved systematic bits are introduced to the input of the inner 


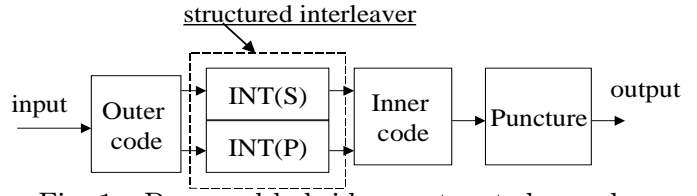

Fig. 1. Proposed hybrid concatenated encoder.

encoder before any of the interleaved parity bits. Since the systematic and parity bits are interleaved separately, there are actually two sub interleaving units (INT(S) and $\operatorname{INT}(\mathrm{P})$ ) within this structured interleaver, each of which is implemented as an S-random interleaver.

Because of the interleaver design, the output codeword can be divided into the four fields shown in Fig. 7. The global systematic output $(\mathrm{So} / \mathrm{Si})$ is comprised of the systematic bits at the output of the inner encoder that correspond to the interleaved systematic output of the outer encoder (i.e. the first half of the inner encoder's systematic output). There are two single parity fields, So/Pi and $\mathrm{Po} / \mathrm{Si}$. The So/Pi field is composed of the parity bits at the output of the inner encoder that are created using the interleaved systematic output of the outer encoder (i.e. the first half of the inner encoder's parity output), while the $\mathrm{Po} / \mathrm{Si}$ field is composed of the systematic bits at the output of the inner encoder due to the interleaved parity output of the outer encoder (i.e. the second half of the inner encoder's systematic output). The double parity field $\mathrm{Po} / \mathrm{Pi}$ is the parity output of the inner encoder which is generated using the interleaved parity output of the outer encoder (i.e. the second half of the inner encoder's parity output).

A family of hybrid codes could be generated by unevenly puncturing the different single- and double-parity fields. For the four distinct fields, the puncturing rate $\mathrm{R}$ subscripted with a field name defines the ratio of the number of deleted bits versus the total number of the bits within that field. The puncturing pattern determines different puncturing rate distributed among those four fields. Thus, the code rate $R_{c}$ of the hybrid codes is determined by $1 / R_{c}=4-R_{P_{o} / S_{i}}-R_{P_{o} / P_{i}}-R_{S_{o} / P_{i}}$. Rate $1 / 3$ codes correspond to a total of $100 \%$ puncturing rate $\left(R_{P_{o} / S_{i}}+R_{P_{o} / P_{i}}+R_{S_{o} / P_{i}}=100 \%\right)$ distributed among fields $P_{o} / S_{i}, P_{o} / P_{i}$, and $S_{o} / P_{i}$.

A set of simulations was performed to gauge the performance of the codes listed in Table I for input words of length 512. In Fig. 2, the pair of curves on the left indicate the performance of a standard PCCC as well as a PCCC generated by puncturing the double-parity output of a SCCC encoder with the structured interleaver. The pair of curves on the right compare the performance of two rate $1 / 3$ SCCC codes. The first code is a standard SCCC code with unstructured interleaver and $50 \%$ of the inner encoder's parity bits regularly punctured, while the second code is a SCCC code with the structured interleaver and $50 \%$ puncturing of both the $\mathrm{So} / \mathrm{Pi}$ and $\mathrm{Po} / \mathrm{Pi}$ fields. In addition, the performance of two rate $1 / 3$ hybrid codes is shown. Hybrid code A is constructed by puncturing $25 \%$ of $\mathrm{Po} / \mathrm{Si}$ and $75 \%$ of $\mathrm{Po} / \mathrm{Pi}$, while hybrid code $\mathrm{B}$ is constructed by puncturing $12.5 \%$ of $\mathrm{Po} / \mathrm{Si}$ and $87.5 \%$ of $\mathrm{Po} / \mathrm{Pi}$. The simulation results confirm that (1) The PCCC that is

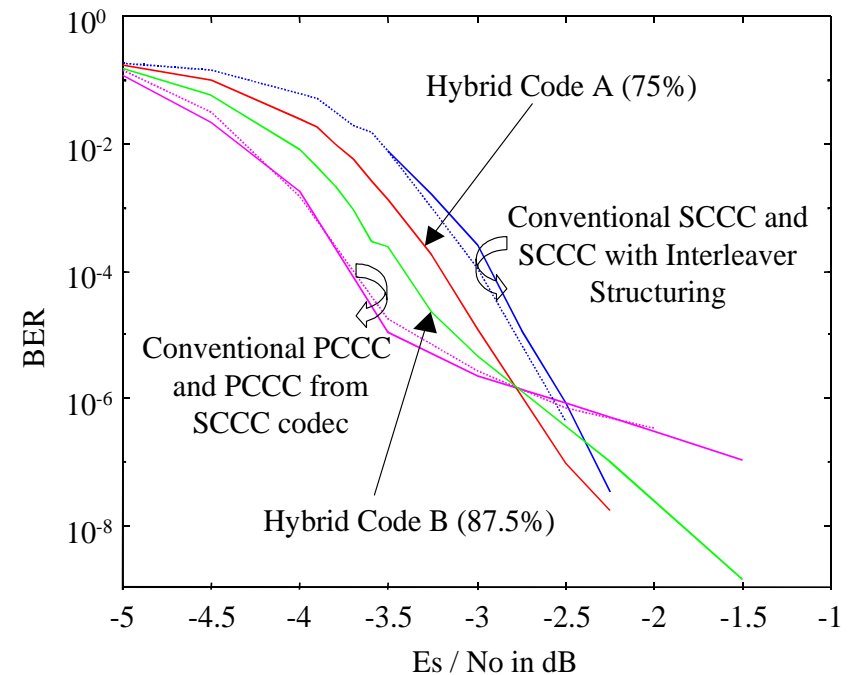

Fig. 2. Performance of rate $1 / 3$ hybrid codes with an input wordlength of 512 bits.

\begin{tabular}{|c||c||c||c||c|}
\hline & $\mathrm{So} / \mathrm{Si}$ & $\mathrm{So} / \mathrm{Pi}$ & $\mathrm{Po} / \mathrm{Si}$ & $\mathrm{Po} / \mathrm{Pi}$ \\
\hline PCCC & $0 \%$ & $0 \%$ & $0 \%$ & $100 \%$ \\
\hline SCCC & $0 \%$ & $50 \%$ & $0 \%$ & $50 \%$ \\
\hline Hybrid code A & $0 \%$ & $0 \%$ & $25 \%$ & $75 \%$ \\
\hline Hybrid code B & $0 \%$ & $0 \%$ & $12.5 \%$ & $87.5 \%$ \\
\hline
\end{tabular}

Table I: Several rate $1 / 3$ hybrid codes characterized by the percentage of bits punctured in each field.

generated by puncturing the SCCC behaves the same as a standard PCCC, (2) The interleaver restriction used by the hybrid encoder does not degrade performance relative to a standard SCCC, and (3) hybrid code A and B split the performance of the rate $1 / 3 \mathrm{PCCC}$ and SCCC. Hybrid code $\mathrm{B}$ has kept more double parity bits, so its performance is closer to that of the PCCC, while code A performs closer to the SCCC because it has punctured more parity bits.

\section{Convergence Analysis}

The convergence of iterative decoding algorithms can be analyzed with the assistance of an extrinsic information transfer (EXIT) chart [4]. Let $X$ denote the binary input to an additive white Gaussian noise (AWGN) channel, $X \in\{ \pm 1\}$, and $Z$ its corresponding output expressed as a log-likelihood ratio (LLR). The soft-input/soft-output (SISO) decoder receives as its input both $Z$ and a priori information $A$, which is also expressed as a LLR. For purposes of the analysis, it is assumed that the sequences $\{A\}$ and $\{Z\}$ at the input to the decoder are infinitely long and that each sequence is i.i.d. Gaussian (conditioned on $X$ ).

With $p_{A}(\cdot)$ denoting the conditional pdf of $\mathrm{A}$ given $\mathrm{X}$, the mutual information $I_{A}=I(X ; A)$ between $X$ and $A$ which can be expressed as

$$
\begin{aligned}
I_{A}= & \frac{1}{2} \sum_{x=-1,1} \int_{-\infty}^{+\infty} p_{A}(\xi \mid X=x) \\
& \cdot \log _{2} \frac{2 p_{A}(\xi \mid X=x)}{p_{A}(\xi \mid X=-1)+p_{A}(\xi \mid X=1)} \mathrm{d} \xi
\end{aligned}
$$

where $0 \leq I_{A}=H(X)-H(X \mid A) \leq H(X) \leq 1$. 
Since $A$ is conditionally Gaussian with variance $\sigma_{A}^{2}$ and mean $\mu_{A}=X \sigma_{A}^{2} / 2$, (1) can be expressed as

$$
I_{A}=1-\int_{-\infty}^{+\infty} \frac{e^{-\left(\left(\xi-\sigma_{A}^{2} / 2\right)^{2} / 2 \sigma_{A}^{2}\right)}}{\sqrt{2 \pi} \sigma_{A}} \log _{2}\left[1+e^{-\xi}\right] \mathrm{d} \xi
$$

Note in the above that there is a one-to-one mapping between the SNR of $A\left(\sigma_{A}^{2} / 4\right)$ and the mutual information $I_{A}[(2)$ is monotonically increasing and hence reversible].

The information at the output of the decoder is denoted $E$, and is characterized by the mutual information $I_{E}=$ $I(X ; E)$. The expression for $I_{E}$ is identical to (1), only with $p_{A}(\cdot)$ replaced with the pdf of $E, p_{E}(\cdot)$. Since $I_{E}$ is dependent on the decoder output, its value is a function of both $I_{A}$ and the channel SNR $E_{b} / N_{o}$ with a functional dependence determined by the code parameters.

Thus, the behavior of a single SISO decoder can be characterized by relating the functions $I_{E}, I_{A}$, and $E_{s} / N_{o}$. A semi-analytic approach can be taken by first fixing the "input entropy" $I_{A}$ (or equivalently, $\sigma_{A}^{2}$ ) and $E_{s} / N_{o}$. Noisy simulated code sequences $\{Z\}$ of long length and SNR of $E_{s} / N_{o}$ are fed into the decoder along with an appropriate randomly generated value of $A$. At the output of the SISO decoder, a histogram is taken to estimate $p_{E}(\cdot)$ which is then used to compute the "output entropy" $I_{E}(\cdot)$ according to (1). Note that $Z, A$, and $E$ could be closely approximated as conditionally Gaussian random variables.

The relationship between $I_{E}$ and $I_{A}$ can then be depicted graphically in an "extrinsic information transfer chart" (or EXIT chart [4]), with a separate curve shown for each value of $E_{s} / N_{o}$. For a concatenated system, two curves can be drawn on the same plot with the output entropy of each encoder becoming the input entropy of the other. Thus a decoding trajectory can be traced by mapping back and forth from one decoder's output entropy to the other decoder's input entropy.

As shown in Fig. 3, four decoding functions are used in the following convergence analysis of the proposed hybrid code. Each function defines an EXIT curve for a particular type of constituent decoder. $Z$ is the channel input which could be further classified as channel systematic input $\left(Z_{-} S\right)$ and channel parity input $\left(Z_{-} P\right) . \quad A_{-} S$ is the extrinsic (i.e. a priori) input for the systematic bits. $E$ is the decoder output which could be further classified as systematic output $\left(E_{-} S\right)$ and parity output $\left(E_{-} P\right)$. All of these inputs and outputs are random variables with entropy defined by (1).

\section{A. EXIT Chart for standard PCCC}

The standard PCCC has two identical rate 1/2 constituent RSC codes. The EXIT curves of its constituent decoders are defined by DEC3. The extrinsic output $\left(E_{-} S\right)$ of the first decoder will be fed into the second decoder as its a priori input $\left(A_{-} S\right)$, and vice versa. The decoding trajectory of the extrinsic information during iterative decoding follows a staircase path along the EXIT curves of the two decoders [4]. Convergence occurs when the two curves no longer overlap, i.e. when a "tunnel" opens up.

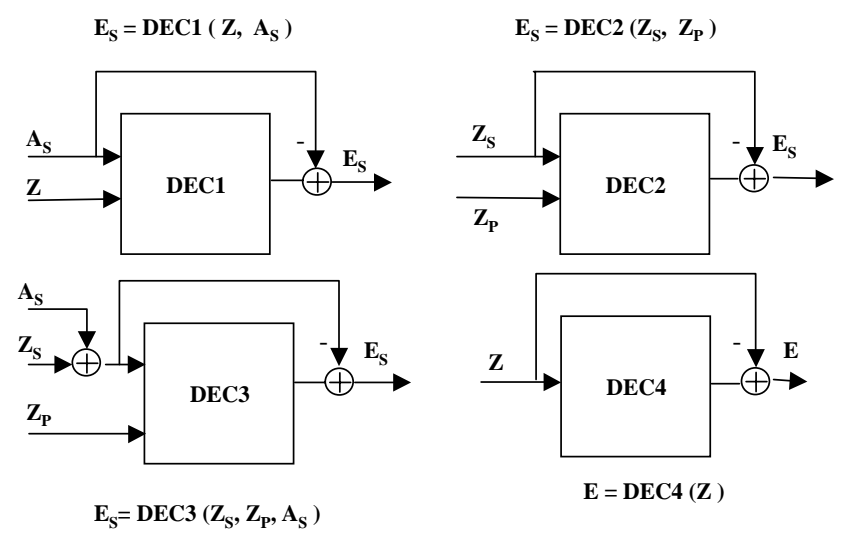

Fig. 3. Four decoding functions used by the EXIT analysis.

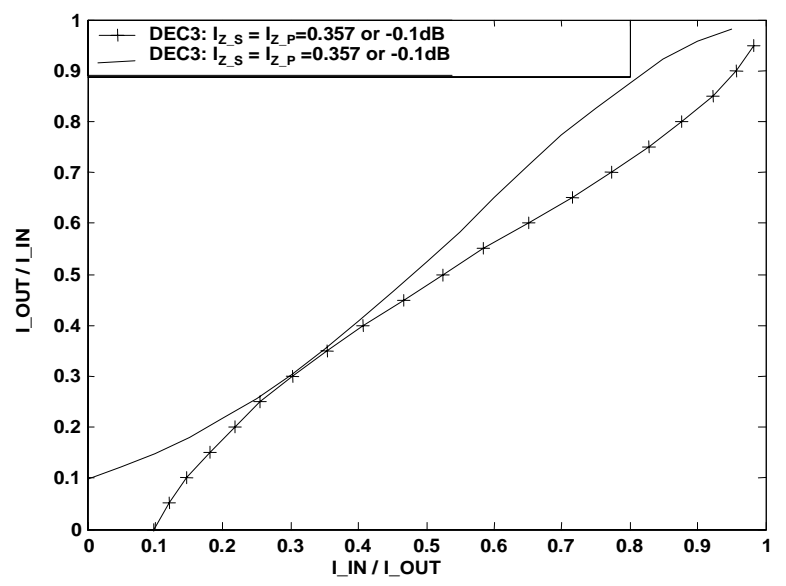

Fig. 4. EXIT chart for a rate $\frac{1}{3}$ PCCC.

Fig. 4 shows an EXIT chart for the rate $1 / 3$ PCCC with generators $(35,23)$ and a frame size of $10^{5}$ bits at a channel SNR of $E_{b} / N_{o}=-0.1 \mathrm{~dB}$. Note that for this SNR, the convergence tunnel just begins to open up.

\section{B. EXIT Chart for standard SCCC}

Consider a standard rate $1 / 3$ SCCC code generated by a rate $1 / 2$ outer RSC code and an identical inner code with $50 \%$ of its parity bits punctured. The inner decoder uses DEC1, while the outer decoder uses DEC4. The DEC1 output $\left(E_{-} S\right)$ is used as the DEC4 input $(Z)$ and the DEC4 output is used as the a priori input $\left(A_{-} S\right)$ of DEC1. Fig. 5 shows an EXIT chart for a rate $1 / 3$ standard SCCC code with constituent RSC $(35,23)$ and frame size of $10^{5}$ bits at a channel SNR of $E_{b} / N_{o}=0.7 \mathrm{~dB}$, which is when the tunnel just begins to open.

\section{Structural equivalence of HCCCs and the proposed hy- brid codes}

The EXIT chart analysis deals with sequence averages. Provided that the PCCC or SCCC encoders are punctured evenly throughout the codeword, the EXIT chart can accurately predict the location of the turbo cliff. However, if the codes are unevenly punctured the precision of the analysis becomes compromised. Thus, an alternative representation for the proposed hybrid code is necessary.

Provided that the frame size is sufficiently long so that edge effects in the decoder (due to tail bits) can be ne- 

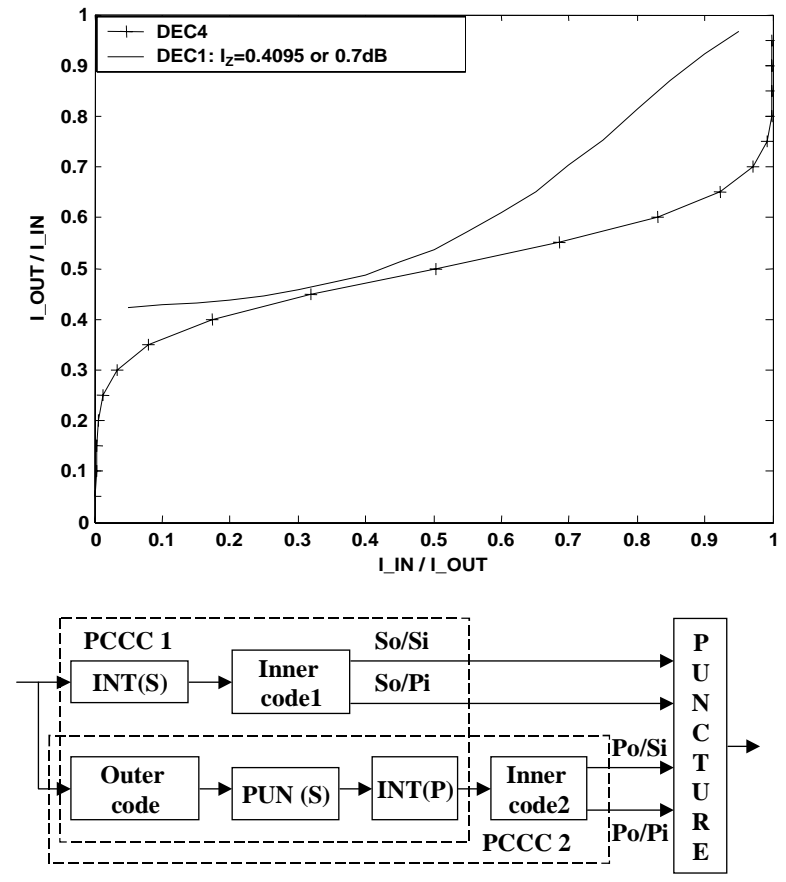

Fig. 6. The proposed hybrid codes as a subclass of HCCCs.

glected, the proposed hybrid code can be considered to be a subclass of HCCCs [3], as illustrated in Fig. 6. In this interpretation, all of the systematic bits at the output of the outer code block are punctured and the parity bits interleaved. The key to the convergence analysis is to recognize that Fig. 6 actually contains two PCCC codes. The codeword of PCCC 1 includes fields: $S o / S i, S o / P i$, and $\mathrm{Po} / \mathrm{Si}$. The codeword of PCCC 2 includes fields $\mathrm{So} / \mathrm{Si}$, $\mathrm{Po} / \mathrm{Si}$, and $\mathrm{Po} / \mathrm{Pi}$. The $\mathrm{So} / \mathrm{Si}$ and $\mathrm{Po} / \mathrm{Si}$ fields are both shared by two PCCCs in Fig. 7. However, while $S o / S i$ is the systematic output for PCCC 1 , it is a parity field for PCCC 2. Likewise, the $\mathrm{Po} / \mathrm{Si}$ field is the systematic field for PCCC 2 and a parity field for PCCC 1.

If the systematic bits $(S(D))$ and parity bits $(P(D))$ of the constituent code have been switched, then its generator polynomials $(g 1(D), g 2(D))$ must also be switched accordingly to preserve the parity check equation of the convolutional code: $P(D) * g 1(D)=S(D) * g 2(D)$. Thus, while both RSC encoders for PCCC 1 use generators $(35,23)$, for PCCC 2 the Inner2 code still uses $(35,23)$ but the Outer2 code uses $(23,35)$ instead. The major advantage of this reconfiguration is that the uneven code puncturing of the proposed hybrid codes are now transformed into an even puncturing pattern within this equivalent HCCC structure and thus the corresponding EXIT chart analysis will be more precise.

\section{EXIT chart analysis for the equivalent $H C C C$}

Because there are two PCCC codes embedded in the HCCC structure of Fig. 6, we now need two EXIT charts to perform the analysis. The iterative decoding within each EXIT chart is called a local loop, while the switching process between the two charts is called a global loop. The
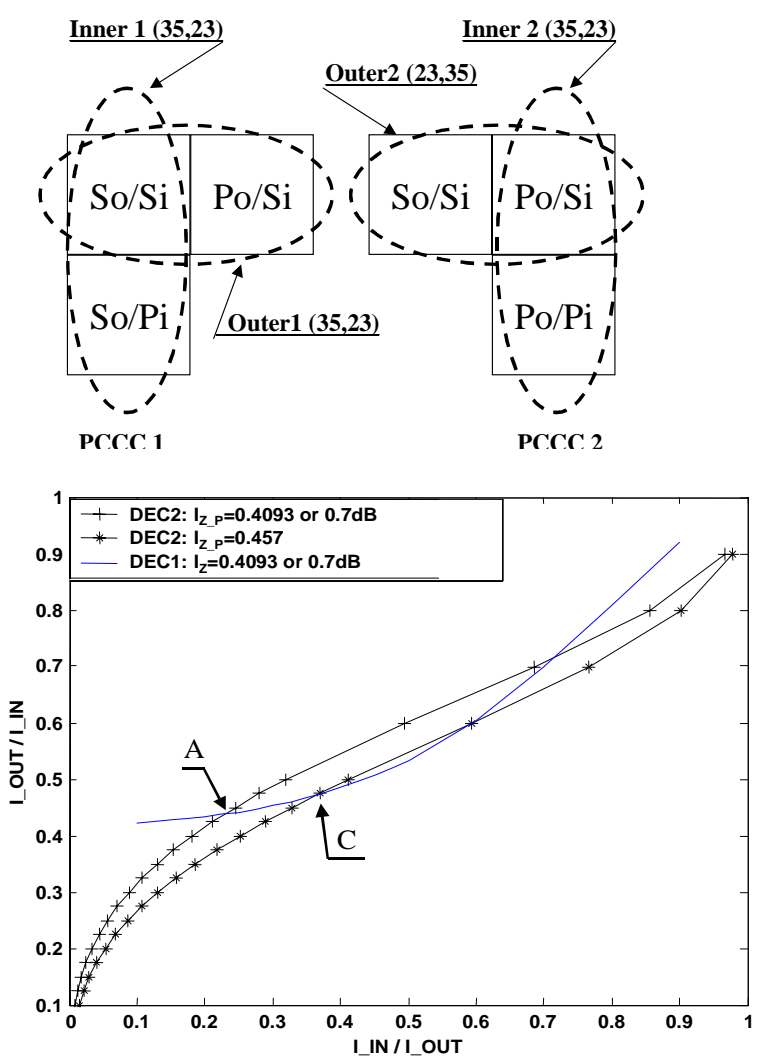

Fig. 8. EXT2 of PCCC2 in rate $\frac{1}{3}$ SCCC.

local loop helps to boost the entropy for fields $\mathrm{Po} / \mathrm{Si}$ and $\mathrm{So} / \mathrm{Si}$, while the global loop helps to skip from a "dead" loop (i.e. a local loop that is stuck at a pinch-off point in one EXIT chart) to a "live" loop (i.e. a local loop in the other EXIT chart that has not yet reached the pinch-off point). By switching back and forth between two EXIT charts, the entropy for $\mathrm{So} / \mathrm{Si}, \mathrm{Po} / \mathrm{Si}$ will grow gradually. Once the entropy for either $\mathrm{Po} / \mathrm{Si}$ or $\mathrm{So} / \mathrm{Si}$ reaches a certain level that the decoding tunnel inside EXT1 or EXT2 opens, then the code will converge.

As an example, the convergence process for a rate $1 / 3$ SCCC code is presented. In its equivalent HCCC structure, both PCCCs have a rate $2 / 3$ punctured RSC code (Inner 1 and Inner 2) and a rate $1 / 2$ RSC code (Outer 1 or Outer 2). For purposes of computing EXIT curves, the Inner1 and Inner2 codes are decoded using DEC1 while the Outer1 and Outer2 codes are decoded using DEC2. In particular, DEC1 feeds its output into DEC2 as $Z_{-} S$ and the DEC2 output is fed into DEC1 as $A_{-} S$.

Figs. 8-9 illustrate the code convergence process when $E_{b} / N_{0}=0.7 \mathrm{~dB}$. The EXIT curves (marked DEC1) for the Inner1 and Inner2 codes are fixed throughout the whole process, because their decoder (DEC1) input $(Z)$ has a fixed channel SNR of $0.7 \mathrm{~dB}$. The decoding process starts using the EXT2 figure. The channel parity input $\left(Z_{-} P\right)$ in DEC2 is first set to the channel SNR of $0.7 \mathrm{~dB}$, in order to define the first EXIT curve (line with ' + ') for Outer2 decoder. The local loop of EXT2 iteratively decodes the Inner2 (DEC1) and Outer2 (DEC2) codes until it hits the first pinch-off point 'A'. The output entropy (0.438) at 'A' 


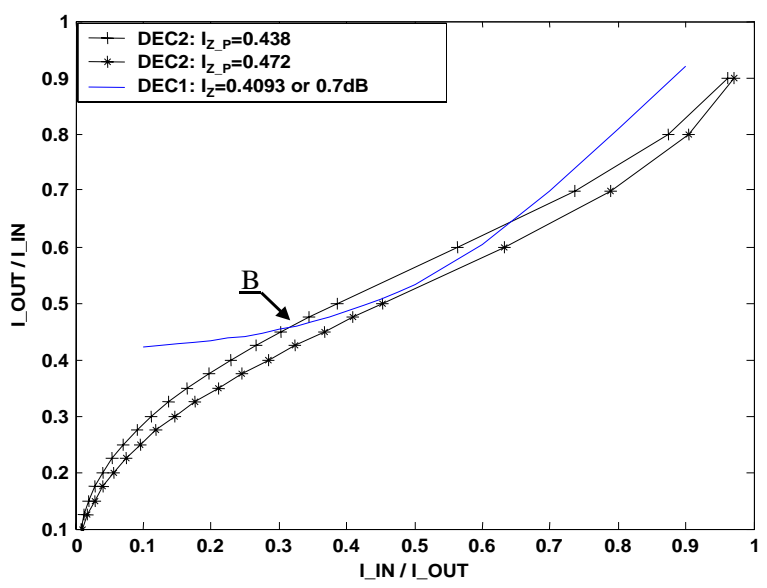

Fig. 9. EXT1 of PCCC1 in rate $\frac{1}{3}$ SCCC.

is the entropy for field $\mathrm{Po} / \mathrm{Si}$ in PCCC2.

At this point, we can switch to the EXT1 chart. Since $\mathrm{Po} / \mathrm{Si}$ is the parity field for Outer1 code (in the PCCC1 encoder), the EXIT curve (line with ' + ') of Outer1 code is generated by setting the entropy of $Z \_P$ in DEC2 (Outer1 decoder) to be the output entropy at point ' $\mathrm{A}$ '. The local loop of EXT1 hits its own pinch-off point 'B'. The output entropy $(0.457)$ at ' $\mathrm{B}$ ' is the entropy for field $\mathrm{So} / \mathrm{Si}$ in PCCC1. Then, we switch back to EXT2. Since So/Si is the parity field for Outer2 code in PCCC2, the second EXIT curve (line with '*') for Outer2 decoder is generated by setting the entropy of $Z_{-} P$ in DEC2 to be the output entropy at point 'B'. Again, the local loop in EXT2 hits a pinch-off point, this time at location ' $\mathrm{C}$ '. When switching to EXT1 again, the second EXIT curve (line with '*') for Outer1 decoder is generated by setting the entropy of $Z \_P$ to be the output entropy at ' $\mathrm{C}$ '. This time, the decoding tunnel between Inner1 and Outer1 decoders opens. Thus the code converges at $0.7 \mathrm{~dB}$, which agrees with the analysis of a standard SCCC (i.e. Fig. 5).

Following this same procedure, the convergence behavior for the other hybrid codes is illustrated shown in Figs. $10-11$ including the rate $1 / 3 \mathrm{PCCC}(-0.1 \mathrm{~dB})$, hybrid code A $(0.4 \mathrm{~dB})$, and hybrid code B $(0.1 \mathrm{~dB})$. Note that when interpretating the $\mathrm{PCCC}$ in terms of the HCCC structure, $\mathrm{PCCC} 2$ reduces to a single RSC code. There is only one global loop during the convergence process of these three hybrid codes, mainly because the severe puncturing on the parity bits of the Inner2 code flattens their EXIT curves in the low to moderate input entropy region where these curves provide almost constant output entropy in EXT2. The local loop in EXT2 is already dead once it hits the first pinch-off, thus can't be reactivated by the global loop. Thus, the convergence behavior of these codes are primarily controlled by the Inner2 code. Moreover, puncturing the systematic bits of Inner2 code will reduce the output entropy for $\mathrm{Po} / \mathrm{Si}$ field, which has a detrimental effect on code convergence.

\section{Conclusions}

The proposed hybrid concatenated encoder is able to generate a range of codes which at one extreme have performance that is identical to a SCCC and at the other extreme

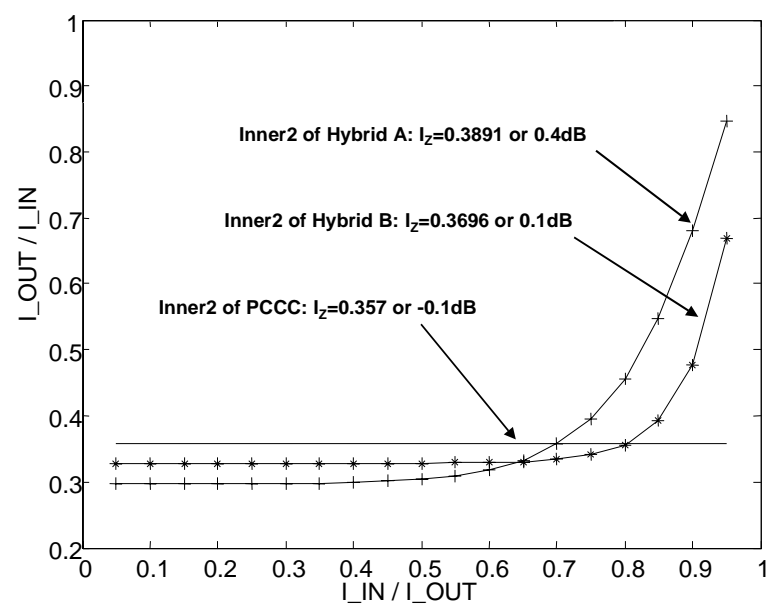

Fig. 10. EXT2 of PCCC2.

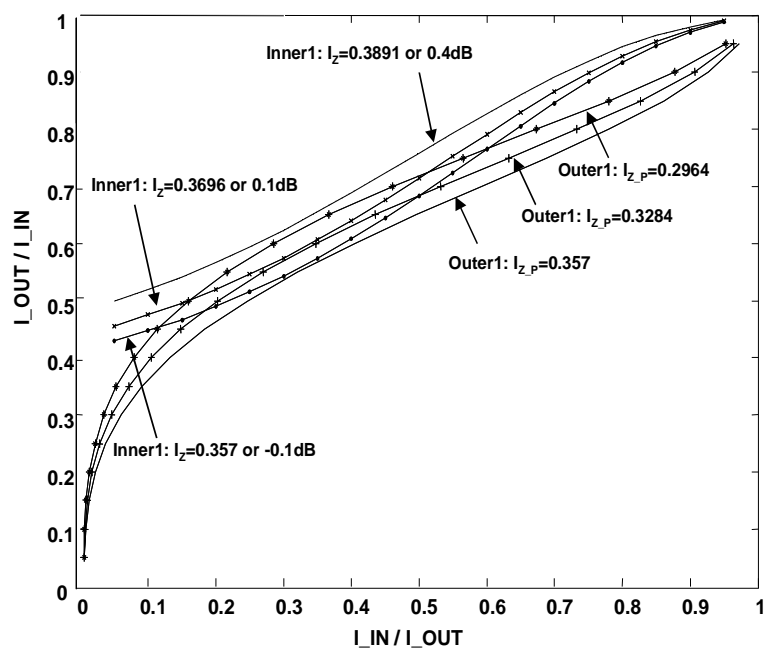

Fig. 11. EXT1 of PCCC1.

performance identical to a PCCC. The encoder structure itself is merely a SCCC encoder with a structured interleaver and puncturing pattern. In order to predict the location of the turbo cliff, it's necessary to remodel the proposed code as a pair of PCCCs, due to the non-constistant puncturing pattern throughout the codeword. While some insight has been given regarding the design of hybrid concatenated codes, future work should focus on more formal design rules for hybrid concatenated codes.

\section{REFERENCES}

[1] Y. Wu and M. Valenti, "An ARQ technique using related parallel and serial concatenated convolutional codes," in Proc., IEEE Int. Conf. on Commun., vol. 3, (New Orleans, LA), pp. 1390-1394, June 2000.

[2] N. Chandran and M. C. Valenti, "Bridging the gap between parallel and serial concatenated codes," in Proc., Virginia Tech Symp. on Wireless Personal Commun., pp. 145-154, June 2002.

[3] D. Divsalar and F. Pollara, "Serial and hybrid concatenation codes with applications," in Proc., Int. Symp. on Turbo Codes and Related Topics, (Brest, France), pp. 80-87, Sept. 1997.

[4] S. ten Brink, "Convergence behavior of iteratively decoded parallel concatenated codes," IEEE Trans. Commun., vol. 49, p. 17271737 , Oct. 2001. 